\title{
Maximum Power Point Tracking and Harmonic Reducing Control Method for Generator-Based Exercise Equipment
}

\author{
Meng-Hui Wang ${ }^{1}$, Mei-Ling Huang ${ }^{2, *}$ and Wei-Jhe Jiang ${ }^{1}$ \\ 1 Department of Electrical Engineering, National Chin-Yi University of Technology, No.57, Section 2, \\ Chung-Shan Road, Taiping District, Taichung City 41170, Taiwan; wangmh@ncut.edu.tw (M.-H.W.); \\ f127860@gmail.com (W.-J.J.) \\ 2 Department of Industrial Engineering \& Management, National Chin-Yi University of Technology, \\ No.57, Section 2, Chung-Shan Road, Taiping District, Taichung City 41170, Taiwan \\ * Correspondence: huangml@ncut.edu.tw; Tel.: +886-4-2392-4505 (ext. 7653); Fax: +886-4-2393-4620
}

Academic Editor: Akhtar Kalam

Received: 11 November 2015; Accepted: 2 February 2016; Published: 11 February 2016

\begin{abstract}
This study uses a sliding mode control (SMC) in a generator-based exercise equipment (GBEE) with nonlinear $P-V$ characteristic curves. A $P-V$ characteristics curve can be influenced by varying the pedaling speed of the generator. The traditional maximum power point tracking (MPPT) control method is used with perturb and observe algorithms ( $\mathrm{P} \& \mathrm{O})$, extremum seeking control (ESC), etc. However, these control methods are not robust enough for control. SMC is created by two pattern methods for robustness control, approaching and sliding conditions. However, SMC allows infinite high-frequency switching of the sign function. If the sign function is used to switch the converter, it will cause the converter and switch life to be cut short, and also to form high frequency noise. Therefore, this study proposes an extension theory for an intelligent control method that will effectively improve conversion efficiency and responsiveness. This study compares generator input current waveforms for fast Fourier transform (FFT) for three different control methods. Finally, using simulation validates the stability and FFT analysis with power simulation (PSIM) software. The results of upgrading overall efficiency are about a 5\% increase in efficiency and a faster response speed of about $0.5 \mathrm{~s}$. The amount of generator input current harmonic is greatly reduced.
\end{abstract}

Keywords: maximum power point tracking (MPPT); sliding mode control (SMC); extension theory

\section{Introduction}

Advances in science and technology have brought economic growth, as well as great consumption of many resources, oil shortage, and greenhouse effect. Development of new clean energy and relevant technologies is thus urgent. The topic of develop a new green energy is important for the world in recent years. The generator-based exercise equipment (GBEE) are $\mathrm{CO}_{2}$ emission free, inexhaustible and the cleanest of all green energy, have become a topical subject. The GBEE considered here consists of a brushless direct current (DC) generator (BLDCG), three phase bridge rectifiers, power converters, etc. The GBEE converts kinetic energy into electrical energy through a BLDCG. Next, alternating current (AC) power is converted by a rectifier to DC. The DC voltage is transformed and exported by the DC converter which is aboost device that can be used for the conversion of electrical energy from different permanent magnet BLDCG systems. Comparisons with the various BLDCG electric energy conversions are available in the literature [1-5].

GBEE irregular operation may result in unstable power output. Different characteristic curves have different optimal operating points; the optimal operating point is the maximum power point 
(MPP). The differences in the new energy source, when compared to traditional maximum power point tracking (MPPT) control, are in the methods common to the perturb and observe algorithm (P\&O) [6], extremum seeking control (ESC) [7], etc. These methods are easier and have lower costs, but they do not have robustness of control. At the steady state these methods reach, the MPP is proportional to the disturbance. To overcome the above drawbacks, another method of sliding mode control (SMC) for the GBEE is proposed. SMC is based on a nonlinear $P-V$ characteristic curve, defined as: $\partial P / \partial V=0$ of the generator. SMC is caused by two pattern methods for robustness control: approaching and sliding conditions, respectively. Approaching conditions let the system's trajectory reach the sliding surface at the effective time, and sliding conditions limit it to the sliding surface on the system. The final approach to the target is the MPP. If the operating point falls in the MPP of the left or right, it will move in the opposite direction toward the MPP. But SMC allows for an infinite high-frequency switching of the sign function. When the sign function is used to switch the converter, it causes the converter and switch life to be cut short and also to create high frequency noise. Therefore, many scholars have suggested that an intelligent control method for reducing sign function is needed. Fuzzy theory [8], extension theory [9], etc., have frequently been used when searching for an intelligent control method.

This paper proposes an extension SMC (ESMC) that effectively reduces the high frequency switching sign function and effectively reduces the harmonic component of input current. By applying the extension method, the gain constant $\sigma$ could be adjusted to avoid converter component failure from high speed switching as well as high frequency noise interference to the system. The power harmonic component appears when the input of a three-phase bridge rectifier is connected to the generator AC power line. While the DC voltage is hard on the capacitor, it allows high frequency switching on the DC converter output to the load. Finally, this paper uses the power simulation (PSIM) software to simulate the proposed control method and compare the different control methods in the GBEE. Simulation and experimental results indicate that the proposed ESMC more effectively reduces the harmonic components of generator output.

\section{Brushless Direct Current Generator Dynamic Modeling}

The GBEE uses a three-phase BLDCG. The BLDCG equivalent circuit is given in Figure 1. The $R_{\mathrm{S}}$ is the resistance of the coil; $L$ is the self-inductance of the coil; $e_{\mathrm{a}}, e_{\mathrm{b}}$, and $e_{\mathrm{c}}$ represent armature reaction electromotives; $i_{\mathrm{a}}, i_{\mathrm{b}}$, and $i_{\mathrm{c}}$ are the phase currents; and $V_{\mathrm{a}}, V_{\mathrm{b}}$, and $V_{\mathrm{c}}$ are the terminal voltages, and $V_{\mathrm{ab}}$ is the line to line voltage between a-phase and b-phase. The BLDCG equation of state is represented as follows [10]:

$$
\left[\begin{array}{c}
V_{\mathrm{a}} \\
V_{\mathrm{b}} \\
V_{\mathrm{c}}
\end{array}\right]=\left[\begin{array}{c}
e_{\mathrm{a}} \\
e_{\mathrm{b}} \\
e_{\mathrm{c}}
\end{array}\right]-\left[\begin{array}{ccc}
R_{\mathrm{s}} & 0 & 0 \\
0 & R_{\mathrm{s}} & 0 \\
0 & 0 & R_{\mathrm{s}}
\end{array}\right]\left[\begin{array}{c}
i_{\mathrm{a}} \\
i_{\mathrm{b}} \\
i_{\mathrm{c}}
\end{array}\right]-\left[\begin{array}{ccc}
L & 0 & 0 \\
0 & L & 0 \\
0 & 0 & L
\end{array}\right] \frac{\mathrm{d}}{\mathrm{d} t}\left[\begin{array}{c}
i_{\mathrm{a}} \\
i_{\mathrm{b}} \\
i_{\mathrm{c}}
\end{array}\right]
$$

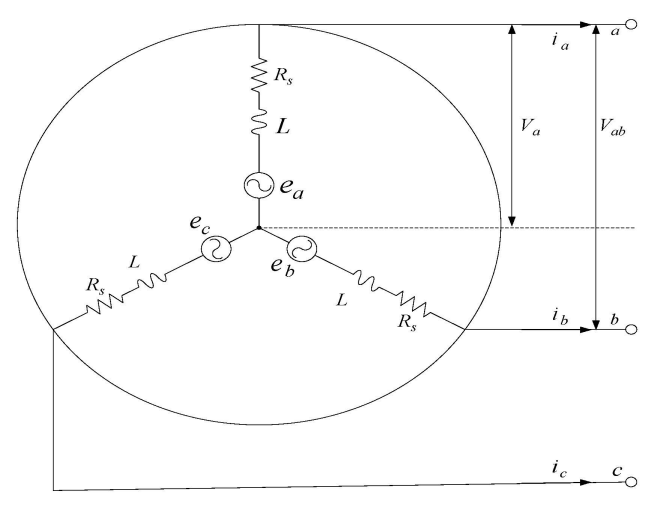

Figure 1. Generator equivalent circuit. 
The relationship between generator internal electromotive $b$ and current of the converter control defines a three-phase commutation function $S_{a}(t), S_{b}(t)$, and $S_{c}(t)$ as Equations (2)-(4) [11]:

$$
\begin{gathered}
S_{\mathrm{a}}(t)=\sum_{n=0}^{\infty}\left[u\left(\omega_{\mathrm{e}} t-2 n \pi\right)-u\left(\omega_{\mathrm{e}} t-\frac{2 \pi}{3}-2 n \pi\right)-u\left(\omega_{\mathrm{e}} t-\pi-2 n \pi\right)+u\left(\omega_{\mathrm{e}} t-\frac{5 \pi}{3}-2 n \pi\right)\right] \\
S_{\mathrm{b}}(t)=S_{\mathrm{a}}\left(t-\frac{2 \pi}{3 \omega_{\mathrm{e}}}\right) \\
S_{\mathrm{c}}(t)=S_{\mathrm{a}}\left(t-\frac{4 \pi}{3 \omega_{\mathrm{e}}}\right)
\end{gathered}
$$

In Equations (2)-(4), $u(t)$ is expressed as a unit step function, and $n$ is an integer, $\omega_{\mathrm{e}}$ is angular velocity of generator rotor. Equation (5) is to define the equivalent generator DC armature current $i_{\mathrm{eq}}(t)$ observing the distribution of three-phase current as follows:

$$
i_{\mathrm{eq}}(t)=\frac{1}{2}\left[S_{\mathrm{a}}(t) S_{\mathrm{b}}(t) S_{\mathrm{c}}(t)\right]\left[i_{\mathrm{a}}(t) i_{\mathrm{b}}(t) i_{\mathrm{c}}(t)\right]^{\mathrm{T}}
$$

According to the commutation function, armature voltage $e_{\mathrm{eq}}(t)$ and voltage across $V_{\mathrm{eq}}(t)$ of BLDC generator are expressed in Equations (6) and (7), respectively:

$$
\begin{aligned}
e_{\mathrm{eq}}(t) & =\frac{1}{2}\left[S_{\mathrm{a}}(t) S_{\mathrm{b}}(t) S_{\mathrm{c}}(t)\right]\left[e_{\mathrm{a}}(t) e_{\mathrm{b}}(t) e_{\mathrm{c}}(t)\right]^{\mathrm{T}} \\
V_{\mathrm{eq}}(t) & =\frac{1}{2}\left[S_{\mathrm{a}}(t) S_{\mathrm{b}}(t) S_{c}(t)\right]\left[V_{\mathrm{a}}(t) V_{\mathrm{b}}(t) V_{\mathrm{c}}(t)\right]^{\mathrm{T}}
\end{aligned}
$$

The equivalent DC dynamics equation of the BLDCG can be obtained by Equation (8):

$$
V_{\mathrm{eq}}=e_{\mathrm{eq}}-R_{\mathrm{s}} i_{\mathrm{eq}}-L \frac{\mathrm{d} i_{\mathrm{eq}}}{\mathrm{d} t}
$$

According to dynamic modeling provided above, The $P-V$ curves of the GBEE can be shown in Figure 2. Figure 2 shows the $P-V$ characteristic curve at different speeds and the human power generation produces different MPPs with respect to different speeds.

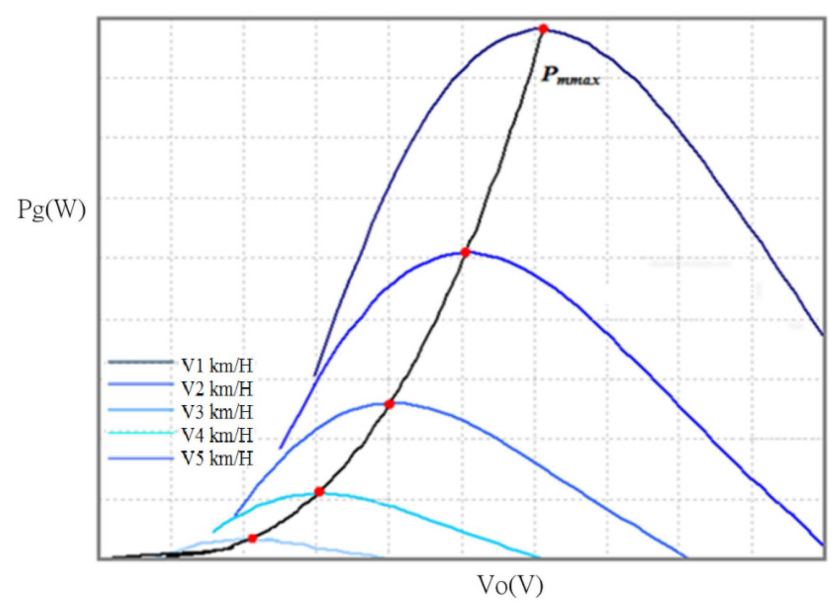

Figure 2. The $P-V$ curves of generator-based exercise equipment (GBEE).

\section{Boost Converter Design}

The power converter of GBEE is a boost converter. The converter is composed of inductors and capacitors, diodes, switches, etc. The boost converter architecture is shown in Figure 3 [12]. 


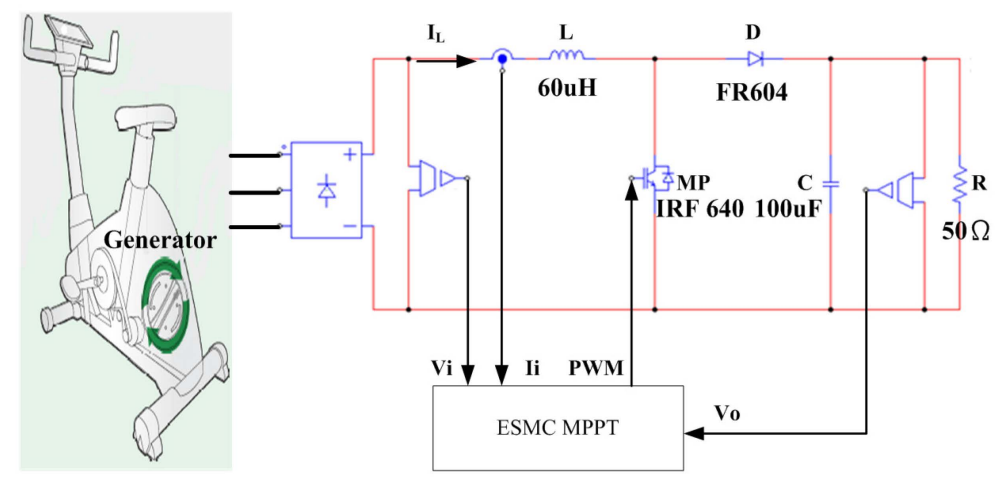

Figure 3. The boost converter architecture for GBEE.

Figure 4 shows the time variation of one cycle switching voltage and inductance current duty cycle ratio. Figure 4 assumes that the current is kept constant in steady state, and the diode and switch have no voltage drop. Therefore, when the switch is on, the switch terminal voltage is $V_{\mathrm{o}}$, the switch off terminal voltage is $V_{\mathrm{o}}, V_{\mathrm{m}}$ is the input voltage of generator terminal voltage after three-phase bridge rectification, and $V_{\mathrm{m}}=V_{\mathrm{eq}}$. The output voltage is expressed as Equation (9), where $t_{\mathrm{on}}$ is turned on, $t_{\text {off }}$ is turned off, one cycle time $T=t_{\text {on }}+t_{\text {off }}$, then Equation (9) is obtained:

$$
\int_{\mathrm{on}} V_{\mathrm{m}} \mathrm{d} t+\int_{\text {off }}\left(V_{\mathrm{m}}-V_{\mathrm{o}}\right) \mathrm{d} t=0, V_{\mathrm{m}} t_{\mathrm{on}}+\left(V_{\mathrm{m}}-V_{\mathrm{o}}\right) t_{\mathrm{off}}=0
$$

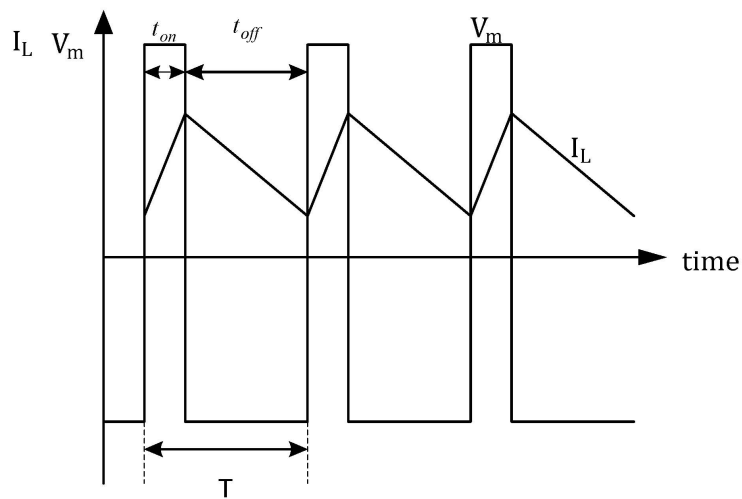

Figure 4. Switching voltage and duty cycle.

By rearrangement of Equation (9), the output voltage and input voltage duty cycle can be rewritten as Equation (10):

$$
\frac{V_{0}}{V_{\mathrm{m}}}=\frac{t_{\mathrm{on}}+t_{\mathrm{off}}}{t_{\mathrm{off}}}=\frac{1}{(1-D)}
$$

The boost converter has two states according to the switch on state in Figure 3:

State 1:

In Figure 3, when the switch $M P$ is turned on, the diode $D$ is turned off, the input voltage flows through the inductor $L$ directly. The inductance current $\dot{I}_{\mathrm{L}}$ rises, and the output end depends on capacitor $C$ which supplies energy to the load $R$. The current $\dot{I}_{\mathrm{L}}$ flows through the switch to form a loop. The voltage and current equations are expressed as Equations (11) and (12):

$$
\dot{I}_{\mathrm{L}\left(\mathrm{MP} \_ \text {on }\right)}=\frac{V_{\mathrm{m}}}{L}
$$




$$
\dot{V}_{\text {o(MP_on })}=-\frac{V_{\mathrm{o}}}{C R}
$$

State 2:

$$
\begin{aligned}
& \dot{I}_{\mathrm{L}\left(\mathrm{MP} \_ \text {off }\right)}=\frac{V_{\mathrm{m}}}{L}-\frac{V_{\mathrm{o}}}{L} \\
& \dot{I}_{\mathrm{O}\left(\mathrm{MP}_{-}\right. \text {off) }}=\frac{I_{\mathrm{L}}}{\mathrm{C}}-\frac{V_{\mathrm{o}}}{C R}
\end{aligned}
$$

Equation (15) represents the dynamic system is $\dot{X}$. By setting the duty cycle is $U$ of the switch by PWM, and substituting Equations (13) and (14) to Equation (15) based on state space averaging method, we have Equations (16) and (17):

$$
\dot{X}=U \dot{X}_{1}+(1-U) \dot{X}_{2}
$$

where $\dot{X}_{1}=\left[\begin{array}{ll}I_{\mathrm{L}\left(\mathrm{MP} \_ \text {on }\right)} & V_{\mathrm{o}\left(\mathrm{MP} \_ \text {on }\right)}\end{array}\right], \dot{X}_{2}=\left[\begin{array}{ll}I_{\mathrm{L}\left(\mathrm{MP} \_ \text {off }\right)} & V_{\mathrm{o}\left(\mathrm{MP} \_ \text {off }\right.}\end{array}\right]$ and duty ratio $U$. Thus the resultant state equations are as follow:

$$
\begin{aligned}
& \dot{I}_{\mathrm{L}}=(U-1) \frac{V_{\mathrm{o}}}{L}+\frac{V_{\mathrm{m}}}{L} \\
& \dot{V}_{\mathrm{o}}=(1-U) \frac{I_{\mathrm{L}}}{\mathrm{C}}-\frac{V_{\mathrm{o}}}{C R}
\end{aligned}
$$

Where the duty ratio denotes the control input. Neglecting the inductors as well as resistors, and assuming $I_{\mathrm{L}}$ as the human power generation current, a non-linear time invariant system is achieved as:

$$
\dot{X}=f(x)+g(x) U
$$

\section{The Proposed Control Method}

\subsection{Sliding Mode Control Mathematical Derivation}

The sliding mode was first found by Russian scholars, it has contributed to the design of multiple system controllers significantly. According to [13,14], the first control step is to designate the sliding surface, then the control rule is designed, so that the system state can be attracted to the sliding surface and it will not deviate from the sliding surface. The system at MPP must satisfy $\frac{\partial P_{\mathrm{m}}}{\partial V_{\mathrm{m}}}=0$, Equation (19) can be obtained [15]:

$$
\frac{\partial P_{\mathrm{m}}}{\partial P_{\mathrm{m}}}=\frac{\partial\left(I_{\mathrm{m}} V_{\mathrm{m}}\right)}{\partial V_{\mathrm{m}}}=I_{\mathrm{m}}+V_{\mathrm{m}} \frac{\partial I_{\mathrm{m}}}{\partial V_{\mathrm{m}}}, \text { and }\left(P_{\mathrm{m}}=P_{\mathrm{g}}, I_{\mathrm{m}}=I_{\mathrm{L}}=i_{\mathrm{eq}}, V_{\mathrm{m}}=V_{\mathrm{eq}}\right)
$$

As shown in Figure 3, the generator provides three-phase bridge rectifier input power. In Figure 4, $V_{\mathrm{m}}$ and $I_{\mathrm{m}}$ are the input voltage and current of the three-phase bridge rectifier at the generator power terminal. Equation (18), defines the sliding surface as in Equation (20):

$$
S=I_{\mathrm{m}}+V_{\mathrm{m}} \frac{\partial I_{\mathrm{m}}}{\partial V_{\mathrm{m}}}
$$

The characteristic curve that we need to control on the sliding surface: $S=0$. From Figure 3 , we can find the $I_{\mathrm{L}}=I_{\mathrm{m}}$ in the circuit. In Figure $5, S$ mode of operation at the MPPT of duty cycle ratio and operating voltage is $\mathrm{u}(0 \leqslant \mathrm{U} \leqslant 1)$, which can be obtained from the PWM duty cycle ratio control switches of the MPP. When $U$ is the PWM controller, the $U$ controller can be obtained, as shown in Equation (22). From Equation (21), we get $S=0$, and according to reference [15], the appropriate 
switchover control law $U_{\mathrm{sw}}=\sigma S$ is selected, $\sigma>0$, considering the controller saturation effect, the control law is Equation (23):

$$
\begin{gathered}
\dot{S}=\left[\frac{\partial S}{\partial X}\right]^{\mathrm{T}}, \dot{X}=\left[\frac{\partial S}{\partial X}\right]^{\mathrm{T}}\left(f(X)+g(X) U_{\mathrm{eq}}\right)=0 \\
U_{\mathrm{eq}}=\frac{\left[\frac{\partial S}{\partial X}\right]^{\mathrm{T}} \cdot f(X)}{\left[\frac{\partial S}{\partial X}\right] \cdot g(X)}=-\frac{\frac{V_{\mathrm{m}}\left(i_{\mathrm{L}}\right)-V_{\mathrm{o}}}{L}}{\frac{V_{\mathrm{o}}}{L}}=1-\frac{V_{m}}{V_{\mathrm{o}}} \\
U= \begin{cases}1 & , U_{\mathrm{eq}}+\sigma S \geqslant 1 \\
U_{\mathrm{eq}}+\sigma S & , 0 \leqslant U_{\mathrm{eq}}+\sigma S<1 \\
0 & , U_{\mathrm{eq}}+\sigma S \leqslant 0\end{cases}
\end{gathered}
$$

Setting $\dot{S}=0$ and the input of $U_{\text {eq, }}$ within the context of MPP and $\sigma S, U$ will track the MPP. Finally, the sliding mode controller designed by the Lyapunov theorem is used for validation; therefore, Equation (24) is required [13,14]:

$$
\dot{V}=S \dot{S}<0
$$

This must satisfy $\sigma>0$ such that the control curve will meet the maximum power on sliding surfaces. However SMC varies in the vicinity of MPP. This study proposes the use of the extension theory to significantly reduce and steady uncertainty, and determine the $\sigma$ values and those for high frequency switching noise suppression.

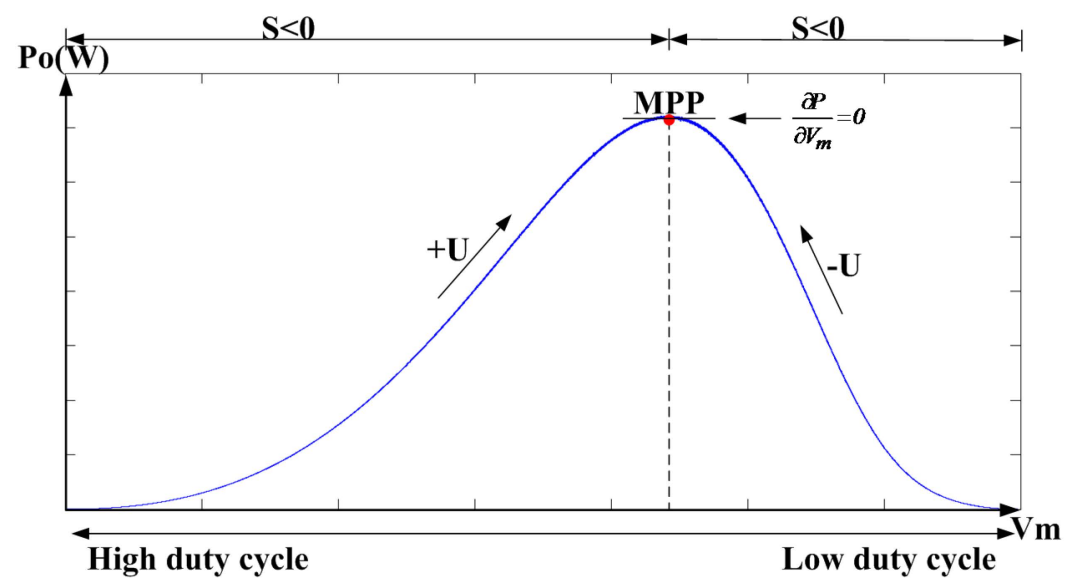

Figure 5. Duty cycle versus operation region.

\subsection{Summary of the Extension Theory}

Extension theory is a new kind of knowledge system based on the concepts of matter-elements and extension sets $[9,16]$. It was first proposed by Cai to solve contradictions and incompatibility problems in 1983 [17]. The hard core of extension theory is two theoretical pillars that include matter-element theory and the theory of extension set. Using extension theory for the proposed control method has six steps leading to evaluation.

Step 1: Determine the classical domains and neighborhood domain and extension matter-element matrix representation method via Equation (25). A matter-element model involves three elements: a name, denoted by $N$; a feature, denoted by $C$; and the value of a feature, denoted by $U$.

$$
R=(N, C, U)
$$


If the matrix eigenvalue is a range, this range is known as classical domain and is included in the neighborhood domain. Assume the classical domain is: $F_{\mathrm{o}}=<a_{k i}, b_{k i}>$, neighborhood domain is: $F_{\mathrm{o}}=<c_{j}, d_{j}>$ and $F_{\mathrm{o}} \in F$. If point $F_{\mathrm{o}}=<c_{j}, d_{j}>$ is the $F_{\mathrm{o}}$ at any point of the domain, the $F_{\mathrm{o}}=<a_{k i}, b_{k i}>$ element of $R_{k}$ can be expressed as in Equation $(26)(j=1,2, \ldots, n)$.

$$
R_{k}=\left[\begin{array}{cccc}
F_{\mathrm{O}} & c_{1} & X_{1} & <a_{k 1}, b_{k 1}> \\
& c_{2} & X_{2} & <a_{k 2}, b_{k 2}> \\
& \vdots & \vdots & \vdots \\
& c_{n} & X_{n} & <a_{k n}, b_{k n}>
\end{array}\right]
$$

where $c_{i}(i=1,2,3, \ldots n)$ are the features in the $F_{\mathrm{o}} ; a_{k i}$ represents the maximum value in the classical domain, and $b_{k i}$ is expressed as the minimum value in the classical domain. Where the $(k=1,2, \ldots, m)$, $(I=1,2, \ldots, n)$. The matter-element corresponding to the neighborhood domain is expressed as follows Equation (27):

$$
R_{p}=\left[\begin{array}{ccc}
F & c_{1} & <c_{1}, d_{1}> \\
& c_{2} & <c_{2}, d_{2}> \\
& \vdots & \vdots \\
& c_{n} & <c_{n}, d_{n}>
\end{array}\right]
$$

where $c_{j}(j=1,2,3, \ldots n)$ are the features in the neighborhood domain $F ; c_{j}$ represents the maximum value in the neighborhood domain, and $d_{j}$ is expressed as the minimum value in the neighborhood domain.

Step 2: Select the $\sigma$ value, so as to reduce the system chattering control law as in Equation (28):

$$
U= \begin{cases}\sigma S & , S \in E \\ U_{\mathrm{eq}} & , S \notin E\end{cases}
$$

When $E$ is the extension region in the extension theory, appropriate control laws are designed for the rational region and the extension region, respectively. After that, the system stability must be ensured. The extension correlation function and the corresponding weights are calculated according to the defined characteristic value $S$.

Step 3: Calculate the degree of association between the data to be measured and the various sets and establish the evaluation degree. The degree of association is established as follows:

$$
k\left(x_{i}\right)= \begin{cases}\frac{-\rho\left(x_{i}, X_{i}\right)}{\left|X_{i}\right|} & , x_{i} \in X_{i} \\ \frac{\rho\left(x_{i}, X_{i}\right)}{\rho\left(x_{i}, X_{\mathrm{p} i}\right)-\rho\left(x_{i}, X_{i}\right)} & , x_{i} \notin X_{i}\end{cases}
$$

where

$$
\begin{gathered}
\rho\left(x_{i}, X_{i}\right)=\left|x_{i}-\frac{a_{k i}+b_{k i}}{2}\right|-\frac{b_{k i}+a_{k i}}{2} \\
\rho\left(x_{i}, X_{\mathrm{p} i}\right)=\left|x_{i}-\frac{x_{j}+d_{j}}{2}\right|-\frac{d_{j}+c_{j}}{2}
\end{gathered}
$$

Step 4: Many studies have discussed methods for selecting the weights in this step. Most of them use an empirical rule and optimization for calculating the weights $W$. This step uses the relationships among weight coefficients to determine the weight percentage of characteristics to the object by:

$$
\sum_{i=1}^{n} W_{i}=1
$$


Step 5: Calculate the relative values $k(q)$ of various grade sets, characteristics value, and the grade value that multiplies and accumulates as follows:

$$
k(q)=\sum_{i=1}^{n} W_{i} k\left(x_{i}\right)
$$

Step 6: By calculating the relative values of degree of association of various grade sets, this paper uses the normalization of Equation (34) to keep the degree of association of each grade set within $<1$, $-1>$. Determine which grade set the matter-element is in. If $K(q)^{*}$ equals 1 , the extension identifies the degree of correlation and the evaluation result as type $q$. The probability of other set types is determined according to the degree of association. This step is used to identify the $\sigma$ value of the controller so as to track the MPP.

$$
k(q)^{*}=\frac{2 k(q)-k(q)_{\max }-k(q)_{\min }}{k(q)_{\max }-k(q)_{\min }}
$$

\section{Harmonic Sources and Harmonic Analysis}

The massive use of power semiconductors in the early 1990s demonstrated that harmonic current through a rectifier and line impedance will generate harmonic voltage drops and create a supply voltage harmonic component. Nonlinear load harmonic is generally determined using an analytical method, simulation, or measurement method. The measurement method is loaded using the analyzer measurements to directly load the current waveform, which is a fast Fourier transform (FFT) direct read out load current spectrum analysis. The simulation method uses software in the load circuits that simulates and calculates the load current waveform and spectrum analysis with computers. The analytical method uses mathematics as a tool for analysis of harmonic current components and effects. Figure 6 shows the generator terminal harmonic current analysis.

According to Figure 6, showing the current waveform of Fourier analysis, $i_{\mathrm{a}}$ current waveform is Fourier series as Equation (35):

$$
\begin{gathered}
i_{\mathrm{a}}=\frac{2 \sqrt{3} I_{\mathrm{D}}}{\pi} \sum_{n=1}^{\infty} \frac{(-1)^{k}}{n} \sin n \omega t, n=6 k \pm 1, k=0,1,2, \cdots \\
=\frac{2 \sqrt{3} I_{\mathrm{D}}}{\pi}\left(\sin \omega t=\frac{1}{5} \sin 5 \omega t-\frac{1}{7} \sin 7 \omega t+\frac{1}{11} \sin 11 \omega t+\frac{1}{13} \sin 13 \omega t \cdots\right)
\end{gathered}
$$

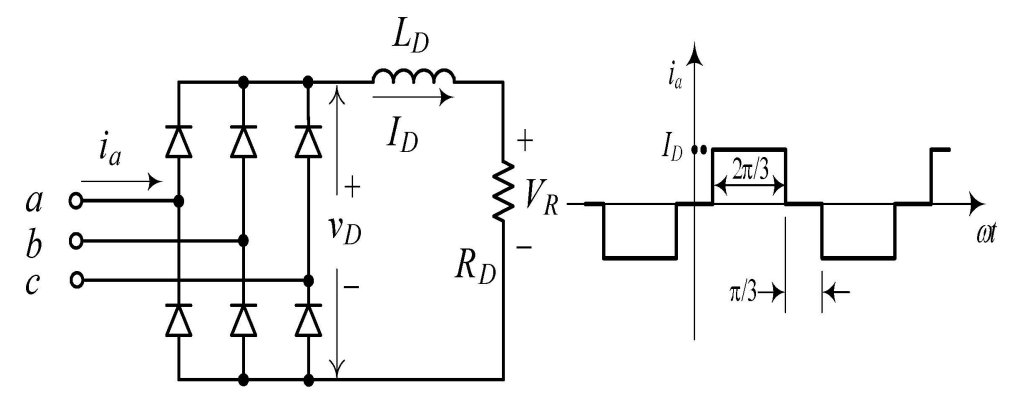

Figure 6. Generator terminal harmonic current analysis.

The definition of total harmonic distortion (THD) rate and THD is composed of multiple harmonic constituents cases, expressed as a percentage of the mean-square value of the harmonic and fundamental frequency ratio of the value effective value. THD of current is defined in Equation (36). $i_{\mathrm{a}}$ is RMS value of fundamental wave current. 


$$
\mathrm{THD} \%=\frac{\sqrt{i_{\mathrm{rms}}^{2}-i_{\mathrm{a}}^{2}}}{i_{\mathrm{a}}}
$$

\section{Simulation}

Table 1 shows the generator specifications of the GBEE. This study uses three control methods with simulation and implementation of the generator specifications in Table 2.

Table 1. GBEE specifications.

\begin{tabular}{ccc}
\hline Items & Specifications & Unit \\
\hline Voltage specification & 220 & $\mathrm{~V}$ \\
Rated power & 200 & $\mathrm{~W}$ \\
Peak power & 500 & $\mathrm{~W}$ \\
Rated speed & 1500 & $\mathrm{rpm}$ \\
Alternator efficiency & $\geqslant 90 \%$ & - \\
Pole P & 4 & $\mathrm{P}$ \\
Armature winding Ra & 6.26 & $\Omega$ \\
Coil inductance Lm & 0.04 & $\mathrm{mH}$ \\
\hline
\end{tabular}

Table 2. Testing results of the proposed control method with different control method. Perturb and observe algorithm: P\&O; sliding mode control: SMC; extension SMC: ESMC.

\begin{tabular}{cccc}
\hline Specifications & P\&O & SMC & ESMC \\
\hline Switching frequency & $100 \mathrm{kHz}$ & $100 \mathrm{kHz}$ & $100 \mathrm{kHz}$ \\
Duty cycle & $0.1-0.9$ & $0.1-0.9$ & $0.1-0.9$ \\
Step size & 0.01 & $\mathrm{~N} / \mathrm{a}$ & $\mathrm{N} / \mathrm{a}$ \\
Gain value $(\sigma)$ & $\mathrm{N} / \mathrm{a}$ & 0.5 & $1-0.2$ \\
\hline
\end{tabular}

PSIM simulates output power and generator voltage characteristic curves for different speeds of GBEE, as in Figure 7. Figure 8a-c illustrates that the simulation results for output power and voltage waveform at the speed of change are $12-15 \mathrm{~km} / \mathrm{h}$ for $\mathrm{P} \& \mathrm{O}, \mathrm{SMC}$, and ESMC, respectively. At speed $7.5 \mathrm{~km} / \mathrm{h}$, as in Figure 9, ESMC possesses the fastest response and highest power output. Table 1 records the simulation results for power output and response speed.

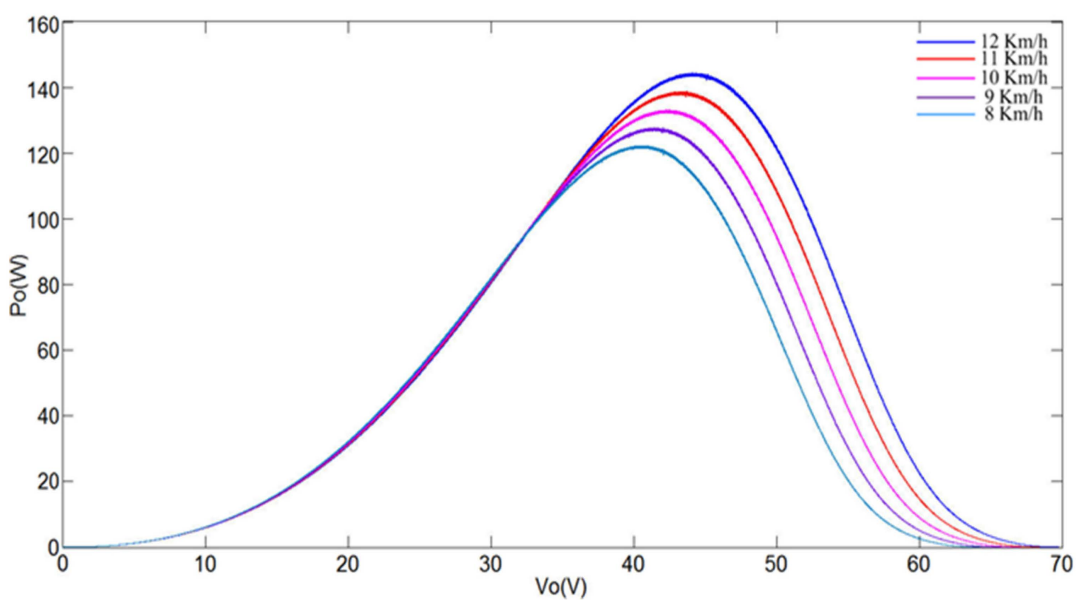

Figure 7. GBEE characteristic curves under different speed.

In this paper, three kinds of control methods are applied to the PSIM simulation of the GBEE. They are the $\mathrm{P} \& \mathrm{O}, \mathrm{ESC}$, and the ESMC. Generator characteristic curve and maximum power output 
of the system are observed. Figure 7 is the characteristic curve diagram of the power output and generator output voltages generated by human power generation at different speeds in the PSIM simulation. Figure 8a-c illustrates the output power and voltage waveforms of the P\&O, ESC, and the ESMC, respectively. Where the speed changes of $12-15 \mathrm{~km} / \mathrm{H}$ during $2.5 \mathrm{~s}$ and $7.5 \mathrm{~s}$ and $15-12 \mathrm{~km} / \mathrm{H}$ during $5 \mathrm{~s}$. Figure 9 shows the comparison of the output power of the three kinds of control methods at the speed of $7.5 \mathrm{~km} / \mathrm{H}$. Figure 9 verifies that the ESMC proposed in this paper possesses the fastest response and highest power output. Table 2 is a comparison of the output power response speed and efficiency of the three kinds of control methods based on Figure 9.

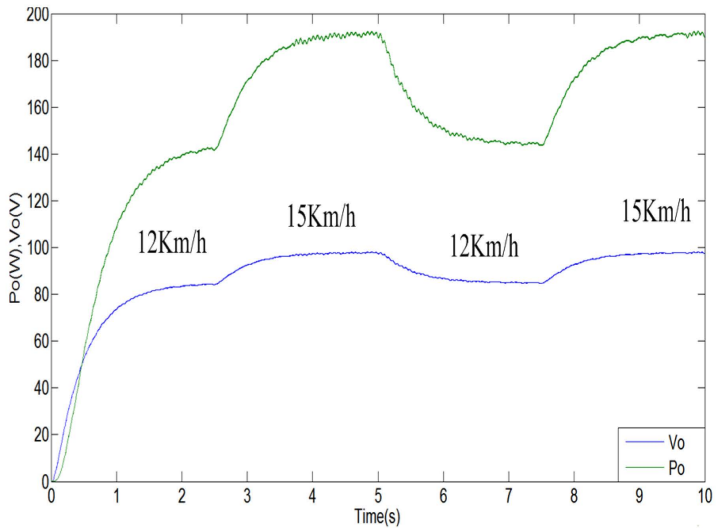

(a)

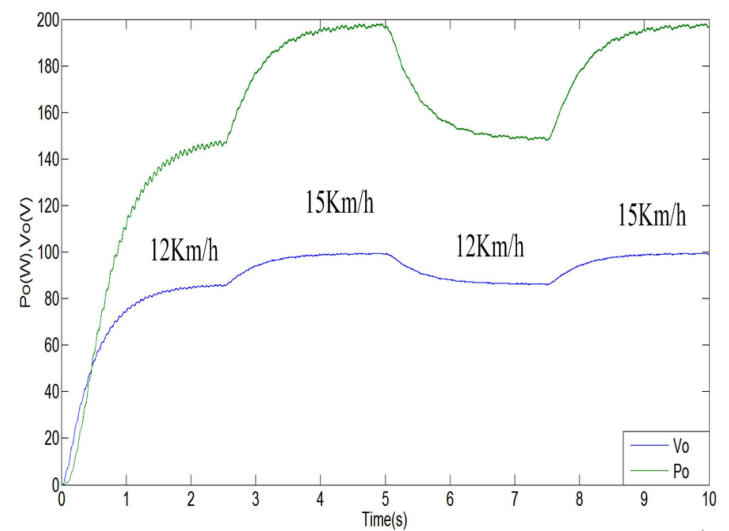

(b)

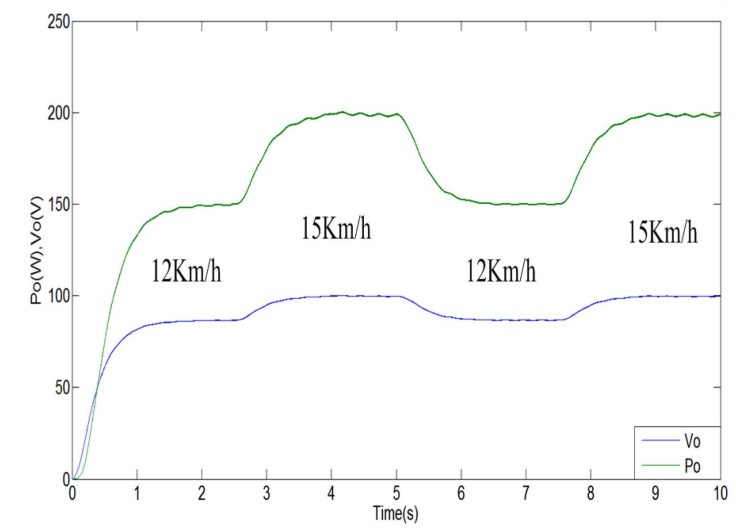

(c)

Figure 8. Simulation results of different control method: (a) P\&O; (b) extremum seeking control (ESC); and (c) ESMC.

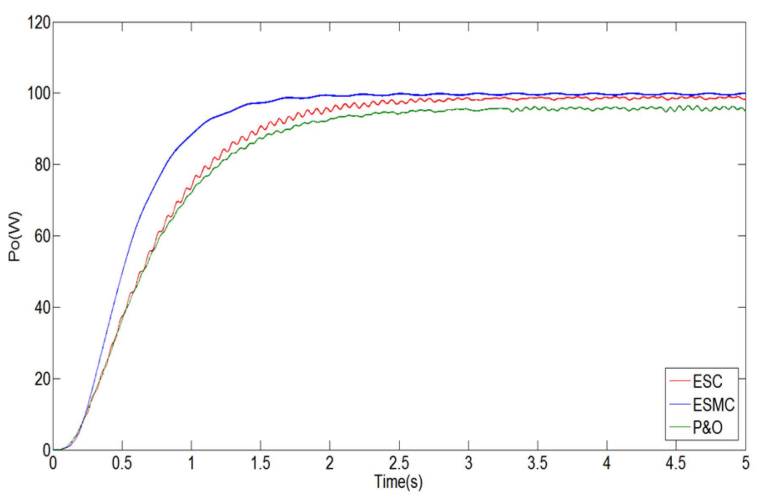

Figure 9. Comparisons of the output power of three control methods. 
Figure 10 is the structural diagram of the actual application. In this paper, Arduino mega 2560 microcomputer control chips were used to apply the ESMC, the P\&O, and the ESC. Arduino I/O pins were used to read the input voltage $V_{\mathrm{i}}$, output voltage $V_{\mathrm{o}}$, input current $I_{\mathrm{i}}$, and output current $I_{\mathrm{o}}$. They were also used to calculate the MPP of the GBEE system. Digital pins of Arduino were used to output PWM signals to the boost converter for conversion, and Arduino was used as the real-time monitoring system to observe the waveform. Figure 11a shows the pedaling speed waveform $\omega$ of GBEE. Figure $11 \mathrm{~b}$ is a comparison of the output power Po for the three control methods. Of the three, the ESMC has the best output power Po and response speed.

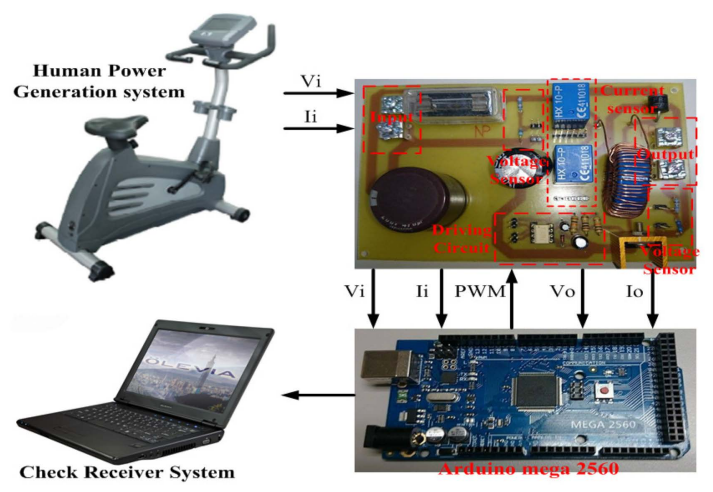

Figure 10. The structural diagram of the actual hardware.

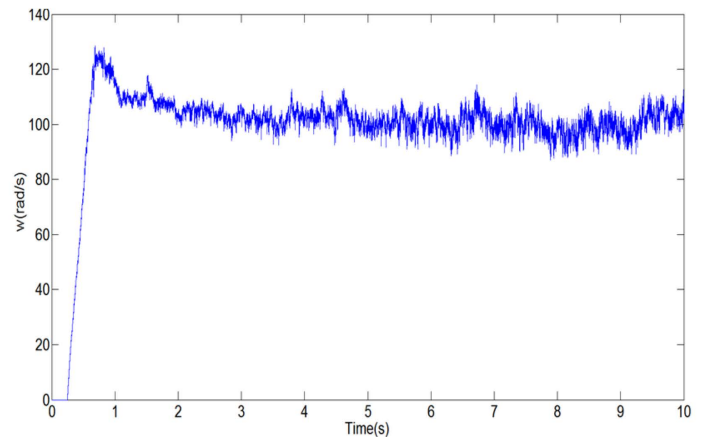

(a)

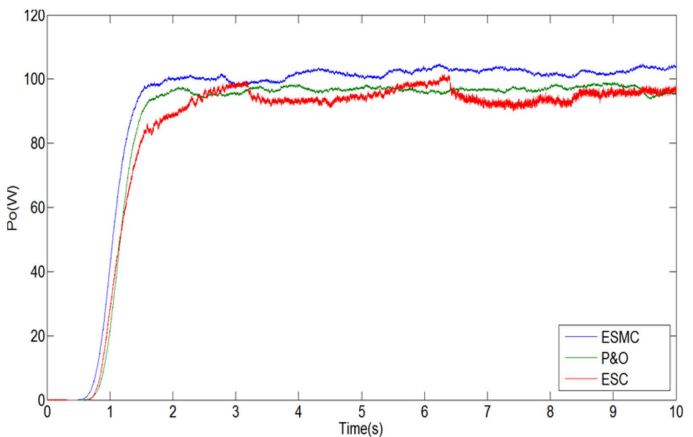

(b)

Figure 11. (a) Pedaling speed waveform $\omega(\mathrm{rad} / \mathrm{s})$; and (b) comparison of the three control methods.

PSIM is used to simulate the input current $i_{\mathrm{a}}$ waveform, and the FFT spectrum analysis of PSIM is applied to the harmonic components of generator input current $i_{\mathrm{a}}$. FFT spectrum analysis of PSIM is applied after the current $i_{\mathrm{a}}$ is obtained. Thus, the FFT spectrum analysis of the voltage regulation control on generator input current $i_{\mathrm{a}}$ can be obtained. Simulate the three control methods of P\&O, ESC, and ESMC with PSIM, respectively, by following the aforementioned method [18]. In this way, the generator input currents $i_{\mathrm{a}}$ of the three control methods can be obtained. Figure 12a shows the relationship between the three control methods and the generator input current $i_{\mathrm{a}}$. Finally, a comparison is made using the FFT spectrum analysis function of PSIM. Figure 12b shows the FFT spectrum analysis of the generator input currents $i_{\mathrm{a}}$ of the three control methods. It can be observed from Figure $12 b$ that the result of ESC is the poorest for the first harmonic component, but the highest for the ESMC and the $\mathrm{P} \& \mathrm{O}$ in the fifth and seventh harmonic components, respectively. It can also be observed from Figure 12b that the harmonic component of the ESMC proposed in this paper is lower than that for both P\&O and ESC. In this paper, the THD is also simulated by PSIM [19]. 


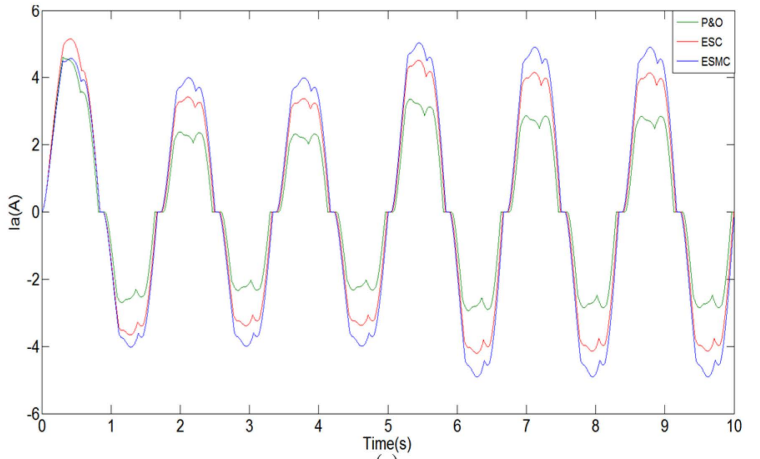

(a)

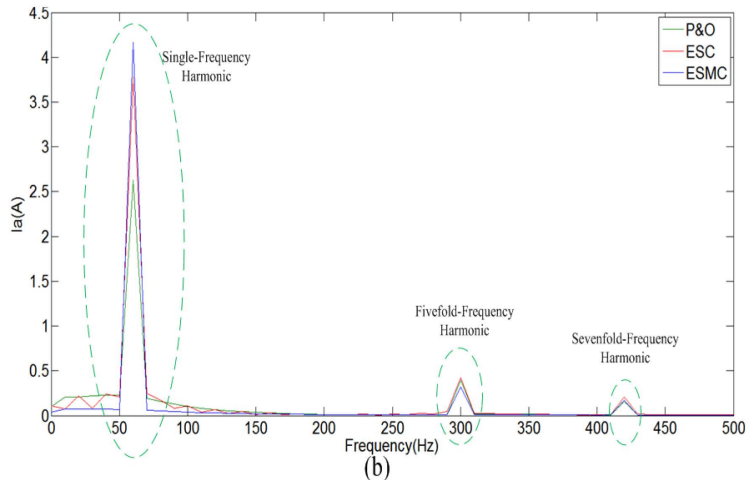

(b)

Figure 12. (a) Three control methods and the generator input current $i_{\mathrm{a}}$; and (b) fast Fourier transform (FFT) spectrum analysis of the generator input currents $i_{\mathrm{a}}$ of the three control methods.

Table 3 shows the THD results for different speeds of the three control methods. The THD of the proposed ESMC is also lower than that for both $\mathrm{P} \& \mathrm{O}$ and ESC.

Table 3. Total harmonic distortion (THD) results for different speeds of the three control methods.

\begin{tabular}{ccc}
\hline Controller Method & Speed & THD $\%$ \\
\hline ESMC & $V=12 \mathrm{~km} / \mathrm{H}$ & $0.895 \%$ \\
ESMC & $V=15 \mathrm{~km} / \mathrm{H}$ & $0.895 \%$ \\
ESC & $V=12 \mathrm{~km} / \mathrm{H}$ & $0.9487 \%$ \\
ESC & $V=15 \mathrm{~km} / \mathrm{H}$ & $0.9487 \%$ \\
P\&O & $V=12 \mathrm{~km} / \mathrm{H}$ & $0.9327 \%$ \\
P\&O & $V=15 \mathrm{~km} / \mathrm{H}$ & $0.9327 \%$ \\
\hline
\end{tabular}

\section{Conclusions}

The simulation results verify that the method proposed in this paper is able to control the nonlinear characteristic behavior of the GBEE at the MPP and enables it to reach and be stabilized at the MPP in the event that the external speed of the generator is changed. The sliding condition calculation mode is used to track the MPP, and the extension theory is applied to select the appropriate key parameter $\sigma$ in designing the parameters of the GBEE and the DC/DC boost converter. The simulation and actual implementation results indicate that with the design MPP of the ESMC, better transient response, and higher output power can be achieved as compared to the design MPP of the ESC and the P\&O. At the end of this paper, the FFT spectrum analysis and THD analysis of input current $i_{\mathrm{a}}$ were explored. It is found that the ESMC can effectively inhibit the harmonic component and that its THD\% is very low and stable.

Acknowledgments: This work was supported in part by the Ministry of Science and Technology, Taiwan, ROC. Under the grant no: 104-2623-E-167-001-ET.

Author Contributions: This study proposes an intelligent control method for the GBEE that can effectively improve conversion efficiency and response speed, and the amount of GBEE input current harmonic is greatly reduced.

Conflicts of Interest: The author declares no conflict of interest.

\section{References}

1. Tan, K.; Islam, S. Optimum Control Strategies in Energy Conversion of PMSG Wind Turbine System without Mechanical Sensors. IEEE Energy Convers. 2004, 19, 392-399. [CrossRef] 
2. Semail, E.; Kestelyn, X.; Bouscayrol, A. Sensitivity of a 5-phase Brushless DC Machine to the 7th Harmonic of the Back-Electromotive Force. In Proceedings of the 2004 IEEE 35th Annual Power Electronics Specialists Conference, PESC 04, Aachen, Germany, 20-25 June 2004.

3. Salameli, I.M.; Kazda, L.F. Analysis of Steady State Performance of the Double Output Induction Generator. IEEE Trans. Energy Convers. 1986. [CrossRef]

4. Salameh, Z.M.; Kazda, L.F. Analysis of the Double Output Induction Generator Using Direct Three-Phase Model Part II-Harmonic Analysis. IEEE Trans. Energy Convers. 1987. [CrossRef]

5. Rahman, M.A.; Zhou, P. Analysis of Brushless Permanent Magnet Synchronous Motors. IEEE Trans. Energy Convers. 2009, 24, 714-724. [CrossRef]

6. Jones, D.C.; Erickson, R.W. Probabilistic Analysis of a Generalized Perturb and Observe Algorithm Featuring Robust Operation in the Presence of Power Curve Traps. IEEE Trans. Power Electron. 2012, 28, 2912-2926. [CrossRef]

7. Bazzi, A.M.; Krein, P.T. Ripple Correlation Control: An Extremum Seeking Control Perspective for Real-Time Optimization. IEEE Trans. Power Electron. 2013, 29, 988-995. [CrossRef]

8. Hsueh, Y.C.; Su, S.F.; Chen, M.C. Decomposed Fuzzy Systems and Their Application in Direct Adaptive Fuzzy Control. IEEE Trans. Cybern. 2014, 44, 1772-1783. [CrossRef] [PubMed]

9. Wang, M.H. Extension Neural Network-Type 2 and Its Applications. IEEE Trans. Neural Netw. 2015, 16, 1352-1361. [CrossRef] [PubMed]

10. Chang, T.Y.; Pan, C.T.; Hsiao, C.C. A Novel Three-Phase Boost Type AC/DC Converter for BLDC Wind Power Generation Systems. In Proceedings of the Taiwan Power Electronics Conference (TWEC), Chiayi, Taiwan, 26-27 May 2010; pp. 144-148.

11. Knshnan, R.; Rim, G.H. Modeling, Simulation an Analysis of Variable Speed Constant Frequency Power Conversion Scheme with A Permanent Magnet Brushless DC Generator. IEEE Trans. Ind. Electron. 1990, 37, 291-296.

12. Mohan, N.; Undeland, T.M.; Robbins, W.P. Power Electronic: Converter, Application and Design; Wiley: New York, NY, USA, 1995.

13. Chu, C.C.; Chen, L.C. Robust maximum power point tracking method for photovoltaic cells: A sliding mode control approach. Sol. Energy 2009, 83, 1370-1378. [CrossRef]

14. Yau, H.T.; Chen, C.L. Fuzzy Sliding Mode Controller Design for Maximum Power Point Tracking Control of a Solar Energy System. Trans. Inst. Meas. Control 2012, 34, 557-565. [CrossRef]

15. Levron, Y.; Shmilovitz, D. Maximum Power Point Tracking Employing Sliding Mode Control. IEEE Circuits Syst. Soc. 2013, 60, 724-732. [CrossRef]

16. Wang, M.H.; Yau, H.T.; Wang, T.Y. Extension Sliding Mode Controller for Maximum Power Point Tracking of Hydrogen Fuel Cells. Abstr. Appl. Anal. 2013. [CrossRef]

17. Cai, W. The Extension Set and Incompatibility Problem. J. Sci. Explor. 1983, 1, 81-93.

18. Zhang, Y.; Zhao, Z.; Yuan, L.; Zhang, H. PSIM and SIMULINK Co-simulation for Three-level Adjustable Speed Drive Systems. In Proceedings of the IEEE 5th International Power Electronics and Motion Control Conference, Shanghai, China, 14-16 August 2006.

19. Hoevenaars, T.; Le Doux, K.; Colosino, M. Interpreting IEEE STD 519d Meeting its Harmonic Limits in VFD Applications. In Proceedings of the IEEE Industry Applications Society 50th Annual Petroleum and Chemical Industry Conference, Houston, TX, USA, 15-17 September 2003; pp. 145-150.

(C) 2016 by the authors; licensee MDPI, Basel, Switzerland. This article is an open access article distributed under the terms and conditions of the Creative Commons by Attribution (CC-BY) license (http://creativecommons.org/licenses/by/4.0/). 